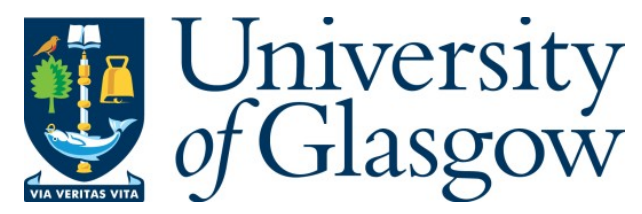

Gallagher, M., McLeod, H. J., and McMillan, T. M. (2016) A systematic review of recommended modifications of CBT for people with cognitive impairments following brain injury. Neuropsychological Rehabilitation, (doi:10.1080/09602011.2016.1258367).

There may be differences between this version and the published version. You are advised to consult the publisher's version if you wish to cite from it.

http://eprints.gla.ac.uk/131552/

Deposited on: 23 November 2016

Enlighten - Research publications by members of the University of Glasgow http://eprints.gla.ac.uk 
Gallagher, M., McLeod, H.J., \& McMillan, T.M. (in press). A Systematic Review of Recommended Modifications of CBT for People with Cognitive Impairments Following Brain Injury.

Neuropsychological Rehabilitation, (accepted 2 November 2016) DOI:

10.1080/09602011.2016.1258367

\section{A Systematic Review of Recommended Modifications of CBT for People with Cognitive Impairments Following Brain Injury}

Melanie Gallagher ${ }^{\text {a }}$

Hamish J. McLeod ${ }^{\mathrm{a}^{*}}$

Thomas M. McMillan ${ }^{a}$

Institute of Health and Wellbeing, University of Glasgow, Glasgow, Scotland

${ }^{a}$ Institute of Health and Wellbeing, College of Medical, Veterinary and Life Sciences, University of Glasgow, 1st Floor, Admin Building, Gartnavel Royal Hospital, 1055 Great Western Road, Glasgow, G12 0XH.

Telephone: 01412110354

melanie.gallagher2@ggc.scot.nhs.uk

hamish.mcleod@glasgow.ac.uk

thomas.mcmillan@glasgow.ac.uk

*Author for correspondence 


\begin{abstract}
Background Due to diverse cognitive, emotional and interpersonal changes that can follow brain injury, psychological therapies often need to be adapted to suit the complex needs of this population.

Aims To synthesise published recommendations for therapy modifications following brain injury from non-progressive traumatic, vascular, or metabolic causes and to determine how often such modifications have been applied to Cognitive Behavioural Therapy (CBT) for post-injury emotional adjustment problems.
\end{abstract}

Method Systematic review and narrative synthesis of therapy modifications recommended in review articles and reported in intervention studies.

Results Database and manual searches identified 688 unique papers of which eight review articles and 16 intervention studies met inclusion criteria. The review articles were thematically analysed and a checklist of commonly recommended modifications composed. The checklist items clustered under themes of: therapeutic education and formulation; attention; communication; memory; and executive functioning. When this checklist was applied to the intervention studies, memory aids and an emphasis on socialising patients to the CBT model were most frequently reported as adaptations.

Conclusions Inconsistent reporting of psychological therapy adaptations for people with brain injury is a barrier to developing effective and replicable therapies. We present a comprehensive account of potential modifications that should be used to guide future research and practice.

Keywords: brain injury; cognitive behavioural therapy 


\section{Introduction}

Brain injury can profoundly impair functioning via effects in cognitive, emotional, sensory, motor and psychosocial domains (Arlinghaus, Shoaib \& Price, 2005). Rates of anxiety and depression in people with brain injury are substantially higher than in the general population (Hiott \& Labbate 2002; Osborn, Mathias, \& Fairweather-Schmidt, 2014; 2015; Scholten et al., 2016), and these are persistent problems with variable recovery trajectories (Arciniegas \& Wortzel, 2014; Hart et al., 2014). In those affected by traumatic brain injury (TBI), anxiety is very common (Osborn, Mathias \& Fairweather-Schmidt, 2016) with up to $60 \%$ meeting criteria for an anxiety disorder (Hibbard, 1998), and more than half experiencing an episode of major depression in the first year following injury (Bombardier et al., 2010). Judd and Wilson (2005) have argued that organic brain damage should be conceptualised and treated in a way that considers the interconnected effects of both organic and psychological consequences of the injury. So, when treating anxiety and depression following brain injury it is expected that existing psychological therapies require adaptation to sensitively respond to organic changes and maximise chances of success (e.g. Gould, Ponsford, Johnston \& Schönberger, 2011). The present review aims to describe and analyse current recommendations for adaptations to Cognitive Behavioural Therapy (CBT) for emotional adaptation in the context of non-degenerative brain damage.

The terms 'acquired brain injury' (ABI) and 'brain injury' are often used interchangeably to describe damage to the brain from diverse causes (Scottish Intercollegiate Guidelines Network [SIGN], 2013; Turner-Stokes, Nair, Sedki, Disler \& Wade, 2011). We will use the term 'brain injury' to cover both terms. Such damage can be focal or diffuse and can vary in severity and location within the brain, leading to a multitude of possible changes in functioning. In terms of cognitive changes alone, symptoms will be dependent on the location and type of brain injury, but can often include changes in memory, attention, processing speed, executive function, and possibly also in awareness of any deficits (Rabinowitz \& Levin, 2014). The World Health Organisation's International Classification of Functioning (WHO ICF) has highlighted this heterogeneity, indicating that every individual affected by brain injury will have a unique set of needs (Wade \& Halligan, 2003). It follows that patients will require psychological therapies that are suitably adapted to meet these diverse needs.

Current treatment guidelines recommend that rehabilitation after brain injury takes place within a holistic neuropsychological multidisciplinary rehabilitation programme, which can address cognitive, emotional and behavioural difficulties to improve engagement 
in meaningful everyday activities (e.g. SIGN, 2013). Amongst the available psychological therapies, CBT has been identified as suitable for treating post-injury depression and anxiety as it is a structured approach focused on concrete thoughts and behaviours (Hodgson, McDonald, Tate and Gertler, 2005). CBT is a "family of interventions" (Hofmann, Asmundson \& Beck, 2013) based on the view that i) cognitive activity affects behaviour; ii) cognitive activity may be monitored and altered and iii) behaviour change may be achieved through cognitive change (Dobson \& Dozois, 2010, p.4). There are elements of CBT that relate to techniques and therapeutic strategies (e.g. exposure to feared stimuli, arousal management skills training) and elements that relate to the content (e.g. developing new beliefs about the self, the world, and the future). Definitions of CBT that differentiate it from other therapies tend to emphasise the negotiation of specific goals, use of active change strategies (e.g. challenging unhelpful beliefs), completion of between session tasks (e.g. homework), and a "here and now" focus, all guided by an individualised formulation derived from the cognitive model (Flach et al. 2015; Tolin, 2010).

Treatment can include components such as assignment of tasks or homework to collect more information about the links between thoughts and action, reflection about experience, and intentional plans to modify thoughts and behaviour, whilst evaluating the adaptiveness of thoughts and behaviour (Dobson, 2013). A meta-analytic review examining the effectiveness of CBT classified a treatment as CBT if it contained any of the following: relaxation training, exposure therapy, behaviour rehearsal (including training in social skills, habit reversal or problem solving), operant procedures (manipulation of reinforcers or punishers for behaviour, including behavioural activation), cognitive restructuring (including direct strategies to identify and alter maladaptive thought processes) (Tolin, 2010). In practice, CBT has been recommended for the treatment of anxiety symptoms following mild-to-moderate traumatic brain injury, as part of a broader neurorehabilitation programme (SIGN, 2013).

But, the evidence-base to inform the adaptation of therapies to treat these heterogeneous needs is very limited. Understanding which techniques should be used to modify psychological therapy for brain injury could improve therapy offered within the holistic, multidisciplinary approach recommended for brain-injury treatment (e.g. SIGN, 2013). To date, there is no systematic review evidence on which to base adaptations to psychological therapies for people affected by brain injury. We addressed this gap by examining the adaptations to CBT needed to treat the common post-injury psychological difficulties of anxiety and depression (Broomfield et al., 2011; Gould, Ponsford, Johnston 
\& Schönberger, 2011). Particular emphasis was placed on the adaptations made to compensate for common cognitive changes following brain injury. The first step was to use existing review articles to develop a checklist of currently recommended modifications to therapy for post-brain injury emotional adjustment. This checklist was then used to systematically analyse current intervention research evidence (from randomised controlled trials and case studies) to determine how many recommended modifications are actually reported in intervention studies. We also identified any additional modifications reported in intervention studies. Finally, the quality of the reporting of treatment fidelity procedures within intervention studies was analysed using an adapted version of the CONSORT checklist (Boutron, Moher, Altman, Schulz, \& Ravaud, 2008).

\section{Methods}

The search strategy was conducted in accordance with the PRISMA statement (Moher, Liberatti, Tetzlaff \& Altman, 2009) in two phases. First, review articles that contained current recommendations for modifying therapy for the brain injury population were identified. Secondly, post-TBI psychological intervention studies were systematically analysed for therapy modifications.

\section{Search Strategy}

The literature search was completed in June 2014 and included online searches of Embase (1980 to 2014 Week 23); Embase Classic (1947-73); Ovid Medline(R) In-Process \& NonIndexed Citations and Ovid Medline(R) (1946-June, 2014); CINAHL (1981-June, 2014); PsycARTICLES (up to June, 2014); Psychology and Behavioural Sciences Collection (up to June, 2014) and PsychInfo (up to June, 2014). Search terms related to brain injury included ((Acquired brain injur*) OR ABI or (traumatic brain injur*) OR TBI OR (brain injur*) OR (head injur*) OR stroke OR CVA); those relating to type of therapy included (CBT OR (behavio*r* therap*) OR (cognitive therap*) OR (cognitive behavio*r* therap*) OR (psycho* therap*) OR psychotherapy*); and mood-related search terms included (depress* OR (low mood) OR (mood disorder*) OR (affective disorder) OR anx* OR OCD OR PTSD OR trauma OR panic OR phobia) ). The search terms for brain injury, type of therapy and mood-related terms were combined using the Boolean operator AND.

Separate inclusion criteria were created for review articles and intervention studies. Review articles were required to a) be a narrative review, systematic review, or other type of review; b) include recommend alterations to cognitive behaviour therapy provided to 
people with brain injury; c) contain recommendations that are specific to CBT or which do not conflict with the CBT model. This meant that review articles discussing third-wave cognitive-behavioural therapies such as Acceptance and Commitment Therapy (ACT) could be included, as these have been built upon CBT, and thus might include alterations relevant to the adaptation of CBT. Intervention studies were required to a) include participants who were aged 16 years and older with a diagnosis of brain injury, either traumatic or non-traumatic, including stroke, hypoxia, ruptured aneurysm or metabolic encephalopathy; b) focus on CBT provided in a one-to-one format with a clinician, thus excluding group or internet-based delivery and delivery as part of a larger cognitiverehabilitation or neuropsychological rehabilitation setting which targeted numerous outcomes; c) include a primary outcome measure of depression, 'low mood,' or anxiety (described as: 'anxiety,' OCD, PTSD, panic disorder, GAD, social anxiety); d) contain a description of the psychological intervention used, including the length of intervention. Third-wave therapy studies such as those using ACT were not eligible for inclusion in the collection of intervention studies as there are therapeutic techniques and proposed mechanisms of change in these approaches that are distinguishable from therapy adaptations made to accommodate cognitive difficulties. Only peer reviewed English language papers journal articles were included.

The reference lists of included studies were manually searched for additional articles that met the review criteria and articles which cited the selected studies were screened for eligibility using the electronic database Web of Science (June, 2014). Finally, when intervention studies mentioned the use of a treatment manual or protocol that could be made available, the authors were contacted and a copy of the treatment manual was requested.

\section{Data extraction and synthesis}

A narrative-synthesis approach is recommended when there is considerable heterogeneity in the included studies in terms of methods, participants and interventions (Popay et al., 2006). This approach was adopted in the present review because of heterogeneity in type and cause of brain injury, types of therapy adaptation, and study design (RCT or single case).

One author (M.G.) extracted data on recommendations for modifications to therapy from the review articles and intervention studies. The stages of the narrative-synthesis approach consisted of: 1) developing a preliminary synthesis 2) exploring relationships 
between articles, and 3) assessing the robustness of the synthesis. This approach followed published guidelines (Mays, Pope \& Popay, 2005; Popay et al., 2006) and was modelled on a previous high-quality narrative synthesis (Leamy, Bird, Le Boutillier, Williams \& Slade, 2011). The data collection process involved 1. Extracting recommended modifications of CBT for brain injury from review articles; and 2. Extracting reported modifications of CBT for brain injury described in intervention studies. Modifications were defined pragmatically (i.e. the study authors explicitly labelled modifications in their description of the treatment) and also they were inferred when there was a discernable alteration to the content or process of the CBT offered (e.g. a deviation from standard CBT content or processes is mentioned by the study authors but not explicitly labelled as a modification). We defined the core processes of CBT as the use of active change strategies (e.g. challenging unhelpful thinking patterns), deliberate use of between session tasks (homework), guided by an individualised formulation based on the cognitive model (Flach et al., 2015). The content parameter was defined by the prioritising of a "here and now" focus over discussion of the past along with an emphasis on building understanding of how thoughts are linked to patterns of behaviour and feeling (David \& Szentagotai, 2006).

\section{Stage 1: Preliminary synthesis}

a) Creation of a data-extraction framework from review articles. Recommended therapy modifications were extracted from each review article. Themes in recommendations were developed using step-by-step guidance on thematic analysis (Braun \& Clarke, 2006). Recommendations from each article were coded. For example, one recommended modification was to provide "psychoeducation to raise patient (and family) awareness of stroke-related cognitive damage' (Broomfield et al., 2011, p. 211). Another indicated that 'clear information about the physical, emotional, and behavioural consequences of the individual's brain injury and mood disturbances is a vital component of therapy and should be provided for both the patient and carers' (Khan-Bourne \& Brown, 2003, p.103). These recommendations were coded as 'stroke-related psychoeducation,' 'involvement of family in psychoeducation' and 'provision of brain-injury related education.' Alongside other recommendations from other papers, the collation of codes led to an overall recommendation 'theme' such as: 'provide clear information/education on effects of brain injury in order to raise awareness and normalise common reactions.'

Once themes within adaptations had been identified, vote counting was used to identify the frequency with which recommended modification themes appeared across all 
articles. If one recommendation-related theme was present in at least two articles, it was added to a data-extraction framework (the Modification-Extraction List). All adaptations within this framework were then grouped using categories informed by 'domains' of cognitive functioning as a preliminary guideline (Lezak, Howieson, Bigler \& Tranel, 2012). This framework therefore provided an overview of recommended adaptations to therapy found in review articles.

b) Preliminary synthesis of intervention studies. A preliminary synthesis of the intervention studies (RCTs and single-case studies) was conducted through tabulation of data, including: study design, sample characteristics, number and duration of treatment sessions, treatment description, and main outcomes.

Stage 2. Exploring relationships between recommended adaptations and reported adaptations

The relationship between recommended modifications recorded on the ModificationExtraction List and reported modifications within intervention studies were explored in a three-step process.

First, the treatment description in each intervention study was examined and adaptations were extracted. Second, the list of these adaptations was matched to the Modification-Extraction List developed during the thematic analysis conducted at Phase 1. The number of matches was summed within intervention articles to provide the total number of adaptations per article, and summed across articles to show which adaptations were most frequently reported in intervention studies. Finally, modifications reported in intervention studies but not included on the Modification-Extraction List were collated. The thematic analysis described in Phase 1 was used to arrive at the final modificationrelated themes.

The overall quality of the reporting of therapeutic interventions was then assessed. This quality assessment was made using an adapted version of the 'treatment' section of the CONSORT checklist extension for non-pharmacologic treatments (Boutron et al., 2008). The adapted scoring scale was as follows:

- Precise details of the experimental treatment were offered (score of 0 indicating information was sparse, 1 indicating main components of treatment were described, and 2 if treatment could be replicated from the description)

- Description of the different components of the intervention was included ( 0 or 1$)$ 
- Description of the procedure for tailoring the intervention to individual participants was present $(0$ or 1$)$

- Details of how the intervention was, or could be standardised were specified ( 0 or 1$)$

- Details of how adherence to the protocol was assessed or enhanced were included (0 or 1$)$

The level of agreement between independent observers using the quality rating and the Modification-Extraction List was determined by a second reviewer rating a subset of the treatment-trial articles $(n=4 ; 25 \%)$. Agreement between the two reviewers was $85 \%$ for quality rating and $86.8 \%$ for the Modification-Extraction List. Disagreements in ratings were resolved by discussion.

\section{Stage 3. Assessing the robustness of the synthesis}

The robustness of the synthesis was judged through using the ratings of agreement described above, and through contacting authors for treatment manuals (when the availability of treatment manuals was alluded to within the article) to determine whether saturation of themes had been reached.

\section{Results}

The flow diagram for the included research and review articles is shown in Figure 1. The search retrieved 755 records, 67 of which were duplicates. The titles and/or abstracts of all remaining 688 studies were reviewed against inclusion/exclusion criteria, and 639 were deemed unsuitable. A total of 23 potentially eligible review articles and 26 intervention studies were identified, of which eight review articles and 12 intervention studies met all inclusion criteria. Following this, four further intervention studies were identified through checking the reference sections of identified articles and through checking studies which had cited the identified articles, providing a total of eight review articles and 16 intervention study articles for review.

\section{INSERT FIGURE 1 HERE}

\section{Stage 1}

a) Creation of a data-extraction framework from review articles 
Summary characteristics of the included review articles can be found in Table 1. The 18item Modification-Extraction List created from analysis of these articles is displayed in Table 2. The modification recommended by the highest number of articles indicated that therapists should provide clear information on the effects of brain injury in order to raise awareness and normalise common reactions (recommended in six articles).

\section{b) Preliminary synthesis of intervention studies}

Information was extracted from the included intervention studies, including study design, sample, treatment protocol and number of modifications from the Modification-Extraction List. The synthesis of intervention studies can be found in Table 3.

INSERT TABLE 1 HERE

Stage 2. Exploring relationships between recommended adaptations and reported adaptations

The variety of adaptations reported across studies were initially mapped onto the Modification-Extraction List to determine which recommended adaptations were most commonly reported by intervention studies. The number of intervention studies that reported each adaptation within the Modification-Extraction List is shown in Table 2; these adaptations are also explored further in the section below.

INSERT TABLE 2 HERE 


\section{Recorded modifications within intervention studies}

One of the most frequently recorded adaptations (eight out of 16 studies) was that the client was educated on the CBT model. This is a core component of CBT and is perhaps why it was so frequently reported. But the emphasis of this adaptation was related to promoting understanding of how specific changes to cognition, affect and behaviour occur as a result of brain injury. It seems that many patients need to learn not only that their cognitive capacities have changed as a result of injury, but also how this gives rise to new patterns of thinking that may substantially influence affect, and behaviour. The use of memory aids such as written notes or audiotapes during the session was also reported in eight studies. This typically took the form of writing down formulations and homework tasks (Gracey, Oldham \& Kritzinger, 2007; Kneebone \& Hull, 2009, Tiersky et al., 2005), writing down coping thoughts on cue cards (Hsieh et al., 2012a, 2012b), and audio-recording of sessions and relaxation exercises (D’Antonio, Tsaousides, Spielman \& Gordon, 2013; Hodgson, McDonald, Tate \& Gertler, 2005; Kneebone \& Jeffries, 2013).

Seven studies described modelling homework completion and generalising homework as adaptations. This included practising homework in session (e.g. beginning exposure work in session; Hodgson et al., 2005; Hsieh at al., 2012a; Kneebone \& Jeffries, 2013), monitoring success of homework activities during the week through recording effects of daily relaxation (Hsieh et al., 2012a, 2012b), providing written instructions to enhance homework compliance (Kneebone \& Hull, 2009), and applying newly learned techniques to daily activities in the home (Tiersky et al., 2005).

Five articles indicated that therapists used concrete examples and helped clients to generate alternative solutions. Examples of these modifications included providing alternative thoughts during cognitive restructuring (e.g. Hsieh et al., 2012a, 2012b, 2012c), and using role-play to rehearse target behaviours (Hsieh et al., 2012a, 2012b, 2012c; Hodgson et al., 2005).

Three studies explicitly reported that the formulation of participants' strengths and weaknesses were based on cognitive assessment. For example, Hsieh et al.'s (2012c) single case study accommodated difficulties with flexibility of thinking by a) recognising that a concrete 'black and white' style of thinking may affect functioning, and b) illustrating key concepts through concrete examples and drawings. One study described using shortened sessions (Hodgson et al., 2005) to accommodate problems 
with attention and one study used longer sessions (90 minutes) to improve time for processing in the initial stage of treatment (Hofer et al., 2013) ${ }^{1}$.

\section{Additional CBT Protocol Modifications Identified in Intervention Studies}

The process of scrutinising the intervention studies revealed modifications not mentioned in existing reviews and therefore not included on the ModificationExtraction List. This reflects an interpretative challenge in the complex intervention literature where standard protocols are modified by adding components from other therapy approaches (Hayes, Long, Levin, \& Follette, 2013). Thus, determining mechanisms of change becomes more challenging when it is unclear whether the modifications reflect an adjustment to standard CBT strategies or are more usefully viewed as an adjunctive treatment addressing mechanisms of change not targeted in standard CBT. We adopted the position that unravelling this complex issue would be helped in the first instance by describe these additional protocol modifications that have not been identified in previous reviews. This will provide a guide for future dismantling studies that seek to specify and test mechanisms of therapeutic change.

Hsieh et al. (2012b, 2012c) augmented their CBT for anxiety following TBI protocol with three sessions of motivational interviewing (MI). They found that CBT was superior to treatment as usual, and that the CBT plus MI condition produced the greatest reduction in anxiety (effect sizes for $\mathrm{CBT}+\mathrm{MI}$ group $=.50$, and for $\mathrm{CBT}+\mathrm{Non}-$ directive counselling group=.24). Tiersky et al. (2005) augmented their CBT protocol with an equal number of cognitive remediation sessions (focused on attention, information-processing and memory). They did not compare the effect of CBT with or without such augmentation, but did find a relatively large treatment effect size of 1.04 compared with a waitlist control group. Hofer et al. (2013) also described a short period of executive skills training in their single case study. This aimed to enhance engagement within CBT by proactively addressing specific deficits in cognitive functioning.

\section{Additional Themes in Modifications Reported in Intervention Studies}

1 An adaptation checklist detailing the specific intervention studies which recorded each adaptation is available from the authors. 
Several studies noted the use of modified diary forms, for example, diary forms which provided examples of common physical sensations associated with anxiety in order to reduce reliance on free recall (Hodgson et al., 2005; Hsieh et al., 2012a, 2012c; Kneebone \& Hull; Lincoln, Flannaghan, Sutcliffe \& Rother, 1997). Common components of CBT were also given greater prominence to support changes after brain injury. For example, some studies highlighted the importance of using personalised metaphors and discussed clients' personal role models, indicating that this may help to reduce load on memory, particularly if someone has difficulty learning new verbal information (Hsieh et al., 2012a, 2012c). Frequent, mid-week prompting to complete homework through telephone calls was also reported (Hodgson et al., 2005; Rasquin, Van De Sande, Praamstra \& Van Heugten, 2009; Tiersky et al., 2005). Clients were guided to choose Specific, Measurable, Realistic, Achievable, and Time Limited (SMART) goals, in order to accommodate executive deficits in planning, abstract thinking, and idea-generation (Hsieh et al., 2012a, 2012c). Finally, six studies noted the need for complex formulations with this population. Such studies suggested that a biopsychosocial model would be appropriate for formulation, due to the reported overlap between psychological maintaining factors and 'physical' brain-injury related maintaining factors in OCD (Hofer et al., 2013), PTSD (King, 2002; Kneebone \& Hull, 2009; McMillan, 1991; McNeil \& Greenwood, 1996) and seizure-related panic after stroke (Gracey et al., 2007). For example, Hofer et al., (2013) described the case of a young man who developed OCD following a traumatic brain injury. The formulation used to guide treatment took account of the lesion site (orbitofrontal region) and neuropsychological effects, a reduced capacity to cope with stressful situations due to emotional changes, and a loss of job and role in life to explain the development of OCD symptoms. They pointed to research which suggests that repetitive behaviour can be associated with both brain injury (e.g. due to memory impairment or perseveration) and OCD, and highlighted the need for good psychological and neuropsychological assessment in order to pinpoint maintaining factors and determine best treatment options.

\section{Quality of treatment reporting}

The CONSORT based appraisal of the quality of the reporting of treatment showed that all articles provided a description of the general components of CBT covered in their interventions (see Table 3). Yet considerable variability was found between studies on 
all other levels of the quality-measurement scale $(n=16$ studies, median quality rating=4, range $=1-5$, ). Single-case studies showed a higher median quality rating (rating=4; $\mathrm{n}=11$ studies) than RCTS (rating=3; $\mathrm{n}=5$ studies). Only two out of the 16 studies measured adherence to treatment.

\section{Stage 3: Assessing the robustness of the synthesis}

Five studies, from sixteen, indicated that a treatment manual was used in their study, and was available. Each of the five intervention trial authors were contacted twice to request manuals with the aim of determining whether the adaptations extracted from the intervention study descriptions reflected the true state of adaptations. Five authors were contacted and none provided the manual (two authors did not reply, one manual was not available in English, one manual was currently being used in another research trial, and one author was unable to locate the manual). Obtaining the original intervention manuals would have helped to determine whether all of the modificationrelated themes had emerged, and thus reached the saturation recommended within narrative synthesis guidance (Mays et al., 2005). The difficulty obtaining manuals points to another area of that needs to improve in order to advance the development of suitably targeted interventions for post-brain injury adjustment.

\section{INSERT TABLE 3 HERE}

\section{Discussion}

This is the first systematic review and narrative synthesis of adaptations made to CBT for depression and anxiety in people with brain injury. Thematic analysis and vote counting were used to develop a checklist based on recommendations in review articles that focused on the psychological treatment of brain injury. Modifications were defined in the terms of reported alterations to the content of CBT (e.g. the deliberate inclusion of information about common changes in capacities following head injury) or alterations in therapeutic technique (e.g. mass repetition of key information). The 18 modifications clustered into five categories: therapeutic education and formulation specific to brain injury; attention, concentration and alertness; communication; memory; and executive functioning. But, when this checklist was used to analyse published intervention studies examining CBT for post-brain injury depression and anxiety it 
became clear that some intervention studies included additional therapy adaptations not reported in previous reviews. Our review also suggests that some of the modifications to CBT process and content for people with brain injury are difficult to distinguish from 'competent' CBT that is being delivered in a dynamic and flexible fashion for people without brain injury. There are also marked variations in the quality of treatment protocol descriptions within intervention studies. Our CONSORT statement derived rating suggests that the description of treatment procedures is generally of higher quality in single-case studies than in RCTs. This highlights the potential of single-case experimental designs (SCEDs) to provide useful information on the development of complex interventions which are adapted to suit this population, and fits with recent guidance on the use of SCEDs within this population (e.g. Evans, Gast, Perdices \& Manolov, 2014). On the other hand, it also draws attention to methodological inadequacies in existing RCTs, and difficulties in carrying out RCTs in a brain injury population are highlighted elsewhere (McMillan, 2013). These findings together emphasise methodological difficulties within this field of study. The mixed quality of reporting of treatment procedures makes it possible that not all therapy adaptations are reported. If this is the case, subsequent attempts to replicate therapeutic outcome when applying the same techniques with their patients will be more likely to fail or result in smaller effects.

\section{Implications for research and practice}

The present review presents a framework within which to understand modifications made to CBT procedures that may circumvent the cognitive deficits that commonly follow brain injury. The systematic approach used here could be a framework for clinicians to apply to their thinking about how to adjust and augment CBT procedures. Developing more systematic ways of reporting adaptations could enhance future braininjury intervention studies. Furthermore, being able to flexibly adapt CBT procedures is a core skill necessary for working with cognitively impaired individuals affected by a wide range of presentations including learning disabilities, pervasive developmental disorders, severe trauma, depression and psychosis (Rossiter \& Holmes, 2013). Thus, improvements in practice guidance in this area may help clinicians working in a wide variety of fields and this could address health inequalities by improving the accessibility of CBT (Rossiter \& Holmes, 2013). 


\section{Limitations}

The diagnostic categories focused on in the present review narrows the generalisation of the findings to adapted CBT for patients whose acquired brain injuries developed following a discrete event, usually sudden in nature, for which rehabilitation might be indicated because of the resultant sudden change to abilities and self-concept. There are several neurodegenerative conditions (e.g. dementia, Multiple Sclerosis) neoplastic diseases (e.g. brain tumour) and infections that also attract psychological treatment need. Our narrowly focused review has highlighted that there is much to learn about how to characterise and quantify CBT adaptations. Future studies could extend this analysis of the literature to include a wider range of aetiologies and disease trajectories. We also recognise that while our Modification-Extraction List was created from a systematic examination of literature, it is not exhaustive and the method of collating all themes for modification requires replication. Furthermore, because the recommended adaptation list was based on review articles alone, examining other sources of information such as books and book chapters might identify further therapy adaptations. Further therapy modifications applied in intervention studies might also have been identified if treatment manuals were available from authors of the studies we reviewed.

There have therefore been limitations in discovering modifications, but the ability of the present article to determine the i) implementation, or ii) effectiveness of such modifications has also been limited. As no treatment manuals were available, and as there was deemed to be poor recording of treatment integrity, it must therefore be highlighted that the modifications within the present article have not been tested, and the present article cannot claim that such modifications are either implemented or effective.

\section{Recommendations for future research}

The present review indicates considerable variability in the use and reporting of CBT adaptations in intervention studies for brain injury. The Modification-Extraction List used here could be extended and refined to improve the precision with which adaptations made to $\mathrm{CBT}$ are reported in future studies. In addition to further testing the reliability of our rating method there may also be benefit in future studies comparing the Modification-Extraction List content to the items covered in the Cognitive Therapy Rating Scale - Revised (CTS-R) (Blackburn, James, Milne, \& Reichelt, 2000) or other measures of CBT fidelity. This would help further ascertain the boundary between 
competently delivered CBT and modified forms more suitable for supporting emotional adjustment following brain injury. This will also help address the observation in the present review that it is sometimes difficult to distinguish between standard and modified forms of CBT from the descriptions in treatment trials.

Much of the material in the present review mainly focused on CBT process modifications that were designed to compensate for cognitive changes following brain injury. However, future research could also more rigorously test the effect of contentbased adaptations, such as the addition of grief work, in order to determine whether this enhances outcomes. The same need to specify and test mechanisms of change applies to emerging therapies such as Acceptance and Commitment Therapy (e.g. Whiting, Simpson, McLeod, Deane \& Ciarrochi, 2012) and Compassionate Mind Training (Ashworth, Gracey, \& Gilbert, 2011). Comparing the effect of these approaches to standard CBT for people with brain injury could provide further information on how the focus of psychological therapy affects outcomes in terms of emotional adjustment and behavioural functioning. All of this points to the need for future studies that take a systematic approach to the development, refinement, and large-scale implementation of complex interventions, for examples, through using UK Medical Research Council guidance (Craig et al., 2008)). This should include careful process evaluation that can guide the real world application of therapies (Moore, Audrey, Barker, Bond, Bonell, et al., 2015a; Moore, Audrey, Barker, Bond, Bonnell, et al., 2015b). Furthermore, only two trials within the present review reported treatment fidelity data, and little information was provided on the training and supervision of therapists. The procedures in place to enhance fidelity are a key mark of quality in psychological treatment trials (Perepletchikova \& Kazdin, 2006) and the quality of these procedures are likely to affect treatment outcomes and implementation capacity (Hogue, Ozechowski, \& Robbins, 2013). The application of these procedures should greatly improve the reporting of future brain injury treatment studies.

The present review also deliberately focusses on individual CBT treatment, and therefore did not evaluate other adaptations that could be applied to psychotherapeutic work. These approaches include the use of technology within therapy (e.g. the use of the Sensecam; Brindley, Bateman \& Gracey, 2011), neurobehavioural approaches (Arco, 2008) or psychotherapy embedded within a large neurorehabilitation programme (Williams, Evans \& Fleminger, 2003; Williams, Evans \& Wilson, 2003). As holistic programmes for treatment have been recommended (McMillan, 2013), such integrative 
and multicomponent studies could provide further insight into adaptations that could fit a wider treatment model that includes but is not restricted to individual therapy. A final point to reiterate is that the analysis of effective therapy modifications should be expanded to include people who have brain damage due to a wider range of acquired, infectious, and neurodegenerative conditions.

\section{Conclusions}

Much remains to be learned about the diverse nature of helping people recover from emotional dysregulation and adaptation following brain injury. Progress in this field will be accelerated if the quality and clarity of required specific therapy adaptations is improved. The modification checklist developed as part of the presented review could improve the capacity of future research to report intervention protocols. Such improvements in precision should lead to more effective and focused interventions that are well suited to the specific needs of people who are struggling to overcome the challenges that commonly emerge in the aftermath of brain injury. 


\section{References}

Arciniegas, D. B., \& Wortzel, H. S. (2014). Emotional and Behavioral Dyscontrol After Traumatic Brain Injury. Psychiatric Clinics of North America, 37(1), 31-53.

*Arco, L. (2008). Neurobehavioural treatment for obsessive-compulsive disorder in an adult with traumatic brain injury. Neuropsychological Rehabilitation, 18(1), 109124.

Arlinghaus, K.A., Shoaib, A.M., \& Price, T.R. (2005). Neuropsychiatric assessment. In J.M. Silver, T.W. McAllister, S.C., \& Yudofsky (Eds.). Textbook of Traumatic Brain Injury. Washington, DC: American Psychiatric Publishing, Inc.

Ashworth, F., Gracey, F., \& Gilbert, P. (2011). Compassion focused therapy after traumatic brain injury: Theoretical foundations and a case illustration. Brain Impairment, 12(2), 128-139.

Blackburn, I.-M., James, I. A., Milne, D. L., \& Reichelt, F. K. (2000). Cognitive Therapy Scale-Revised (CTS-R) (pp. 1-15).

Block, C.K., \& West, S.E. (2013). Psychotherapeutic treatment of survivors of traumatic brain injury: review of the literature and special considerations. Brain Injury, 27 (7-8), 775 - 788.

Bombardier, C.H., Fann, J.R., Temkin, N.R., Esselman, P.C., Barber, J., and Dikmen, S.S. (2010). Rates of major depressive disorder and clinical outcomes following traumatic brain injury. Journal of the American Medical Association. 303, 19381945.

Boutron, I., Moher, D., Altman, D.G., Schulz, K.F., \& Ravaud, P. (2008). Extending the CONSORT statement to randomized trials of nonpharmacologic treatment: explanation and elaboration. Annals of Internal Medicine, 148, 295 - 309. 
Braun, V., \& Clarke, V. (2006). Using thematic analysis in psychology. Qualitative Research in Psychology, 3(2), 77-101.

Brindley, R., Bateman, A., \& Gracey, F. (2011). Exploration of use of SenseCam to support autobiographical memory retrieval within a cognitive-behavioural therapeutic intervention following acquired brain injury. Memory, 19(7), 745-757.

Broomfield, N. M., Laidlaw, K., Hickabottom, E., Murray, M. F., Pendrey, R., Whittick, J. E., \& Gillespie, D. C. (2011). Post-stroke depression: The case for augmented, individually tailored cognitive behavioural therapy. Clinical Psychology \& Psychotherapy, 18(3), 202-217.

Craig, P., Dieppe, P., Macintyre, S., Michie, S., Nazareth, I., \& Petticrew, M. (2008).

Developing and evaluating complex interventions: new guidance. Medical Research Council. Medical Research Council. Retrieved from [http://www. mrc. ac. uk/Utilities/Documentrecord/index. htm? d= MRC004871]

D'Antonio, E., Tsaousides, T., Spielman, L., \& Gordon, W. (2013). Depression and traumatic brain injury: Symptom profiles of patients treated with cognitivebehavioral therapy or supportive psychotherapy. Neuropsychiatry, 3(6), 601-609.

David, D., \& Szentagotai, A. (2006). Cognitions in cognitive-behavioral psychotherapies; toward an integrative model. Clinical Psychology Review, 26(3), 284-298.

Dobson, K.S. (2013). The science of CBT: Towards a metacognitive model of change? Behavior Therapy, 44, 224-227.

Dobson, K.S. \& Dozois, D. (2010). Historical and philosophical bases of the cognitivebehavioral therapies. In K.S. Dobson (Ed.), Handbook of cognitive-behavioral therapies (3rd ed, p3-38). New York: Guilford Press. 
Evans, J.J., Gast, D.L., Perdices, M., \& Manolov, R. (2014). Single case experimental designs: Introduction to a special issue of Neuropsychological Rehabilitation. Neuropsychological Rehabilitation, 1 - 10.

Flach, C., French, P., Dunn, G., Fowler, D., Gumley, A. I., Birchwood, M., Stewart, S.L.K. \& Morrison, A.P. (2015). Components of therapy as mechanisms of change in cognitive therapy for people at risk of psychosis: analysis of the EDIE-2 trial. The British Journal of Psychiatry, 207(2), 123-129.

Gould, K.R., Ponsford, J.L., Johnston, L., \& Schönberger, M. (2001). The nature, frequency and course of psychiatric disorders the first year after traumatic brain injury: a prospective study. Psychological Medicine, 41, 2099-2109.

*Gracey, F., Oldham, P., \& Kritzinger, R. (2007). Finding out if 'the 'me' will shut down: Successful cognitive-behavioural therapy of seizure-related panic symptoms following subarachnoid haemorrhage: A single case report. Neuropsychological Rehabilitation 17(1), 106-119.

Hart, T., Benn, E. K. T., Bagiella, E., Arenth, P., Dikmen, S., Hesdorffer, D. C., et al. (2014). Early Trajectory of Psychiatric Symptoms after Traumatic Brain Injury: Relationship to Patient and Injury Characteristics. Journal of Neurotrauma, 31(7), 610-617.

Hayes, S. C., Long, D. M., Levin, M. E., \& Follette, W. C. (2013). Treatment development: Can we find a better way? Clinical Psychology Review, 33(7), 870882 .

Hibbard, M.R., Uysal, S., Kepler, K., Bogdany, J., and Silver, J. (1998). Axis I psychopathology in individuals with traumatic brain injury. Journal of Head Trauma Rehabilitation, 13, 24-39.

Hiott, D.W. \& Labbate L. (2002) Anxiety disorders associated with traumatic brain injuries. NeuroRehabilitation, 17, 345-55 
*Hodgson, J., McDonald, S., Tate, R., \& Gertler, P. (2005). A randomised controlled trial of a cognitive-behavioural therapy program for managing social anxiety after acquired brain injury. Brain Impairment, 6(3), 169-180.

*Hofer, H., Frigerio, S., Frischknecht, E., Gassmann, D., Gutbrod, K., \& Müri, R.M. (2013). Diagnosis and treatment of an obsessive-compulsive disorder following traumatic brain injury: A single case and review of the literature. Neurocase, 19 (4), $390-400$.

Hofmann, S.G., Asmundson, G.J.G. \& Beck, A.T. (2013). The science of cognitive therapy. Behavior Therapy, 44, 119-212.

Hogue, A., Ozechowski, T. J., \& Robbins, M. S. (2013). Making Fidelity an Intramural Game: Localizing Quality Assurance Procedures to Promote Sustainability of Evidence-Based Practices in Usual Care. Clinical Psychology: Science and Practice, 20(1), 60-77.

*Hsieh, M., Ponsford, J., Wong, D., Schönberger, M., McKay, A., \& Haines, K. (2012a). A cognitive behaviour therapy (CBT) programme for anxiety following moderate-severe traumatic brain injury (TBI): Two case studies. Brain Injury, 26 (2), $126-138$.

*Hsieh, M., Ponsford, J., Wong, D., Schönberger, M., Taffe, J., \& Mckay, A. (2012b). Motivational interviewing and cognitive behaviour therapy for anxiety following traumatic brain injury: A pilot randomised controlled trial. Neuropsychological Rehabilitation, 22(4), 585-608.

*Hsieh, M., Wong, D., Schonberger, M., McKay, A., \& Haines, K. (2012c). Development of a motivational interviewing programme as a prelude to CBT for anxiety following traumatic brain injury. Neuropsychological Rehabilitation, 22 (4), 563-584. 
Judd, D., \& Wilson, S.L. (2005). Psychotherapy with brain injury survivors: An investigation of the challenges encountered by clinicians and their modifications to therapeutic practice. Brain Injury 19, 437-439.

*Kangas, M., \& McDonald, S. (2011). Is it time to act? The potential of acceptance and commitment therapy for psychological problems following acquired brain injury. Neuropsychological Rehabilitation, 21 (2), 250 - 276.

*Khan-Bourne, N., \& Brown, R. G. (2003). Cognitive behaviour therapy for the treatment of depression in individuals with brain injury. Neuropsychological Rehabilitation, 13(1-2), 89-107.

*King, N. S. (2002). Perseveration of traumatic re-experiencing in PTSD; a cautionary note regarding exposure-based psychological treatments for PTSD when head injury and dysexecutive impairment are also present. Brain Injury, 16, 65-74.

*Kinney, A. (2001). Cognitive therapy and brain-injury: Theoretical and clinical issues. Journal of Contemporary Psychotherapy, 31(2), 89-102.

*Kneebone, I. I., \& Hull, S. L. (2009). Cognitive behaviour therapy for post-traumatic stress symptoms in the context of hydrocephalus: A single case. Neuropsychological Rehabilitation, 19, 86-97.

*Kneebone, I. I., \& Jeffries, F. W. (2013). Treating anxiety after stroke using cognitivebehaviour therapy: Two cases. Neuropsychological Rehabilitation, 23(6), 798810.

Leamy, M., Bird, V., Le Boutillier, C., Williams, J., \& Slade, M. (2011). Conceptual framework for personal recovery in mental health: systematic review and narrative synthesis. The British Journal of Psychiatry, 199, 445-452.

Lezak, M.D., Howieson, D.B., Bigler, E.D., \& Tranel, D. (2012). Neuropsychological Assessment (fifth edition). New York: Oxford University Press. 
*Lincoln, N. B., \& Flannaghan, T. (2003). Cognitive behavioral psychotherapy for depression following stroke: A randomized controlled trial. Stroke, 34(1), 111115.

*Lincoln, N. B., Flannaghan, T., Sutcliffe, L., \& Rother, L. (1997). Evaluation of cognitive behavioural treatment for depression after stroke: A pilot study. Clinical Rehabilitation, 11(2), 114-122.

Mays, N., Pope, C., \& Popay, J. (2005). Systematically reviewing qualitative and quantitative evidence to inform management and policy-making in the health field. Journal of Health Services Research and Policy, 10 (1), 6 - 20.

*McMillan, T. M. (1991). Post-traumatic stress disorder and severe head injury. British Journal of Psychiatry, 159, 431-433.

*McMillan, T. (2013). Outcome of rehabilitation for neurobehavioural disorders. NeuroRehabilitation, 32, $791-801$.

*McNeil, J. E., \& Greenwood, R. (1996). Can PTSD occur with amnesia for the precipitating event? Cognitive Neuropsychiatry, 1, 239-246.

Moher, D., Liberati, A., Tetzlaff, J., \& Altman, D.G. (2009). Preferred Reporting Items for Systematic Reviews and Meta-Analyses: The PRISMA Statement. Annals of Internal Medicine, 151(4), 264-269.

Moore, G. F., Audrey, S., Barker, M., Bond, L., Bonell, C., Hardeman, W., et al. (2015a). Process evaluation of complex interventions: Medical Research Council guidance. $B M J, 350: \mathrm{h} 1258$.

Moore, G., Audrey, S., Barker, M., Bond, L., Bonnell, C., Hardeman, W., et al. (2015b). Process evaluation of complex interventions (pp. 1-134). Medical Research Council. Available from https://www.mrc.ac.uk/documents/pdf/mrc-phsrn-processevaluation-summary-guidance/ 
Osborn, A. J., Mathias, J. L., \& Fairweather-Schmidt, A. K. (2014). Depression following adult, non-penetrating traumatic brain injury: A meta-analysis examining methodological variables and sample characteristics. Neuroscience \& Biobehavioral Reviews, 47, 1-15.

Osborn, A. J., Mathias, J. L., \& Fairweather-Schmidt, A. K. (2016). Prevalence of Anxiety Following Adult Traumatic Brain Injury: A Meta-Analysis Comparing Measures, Samples and Postinjury Intervals. Neuropsychology, 30 (2), 247-261.

Perepletchikova, F., \& Kazdin, A. E. (2006). Treatment Integrity and Therapeutic Change: Issues and Research Recommendations. Clinical Psychology: Science and Practice, 12(4), 365-383.

Popay, J., Roberts, H., Sowden, A., Petticrew, M., Arai, L., Rodgers, M., ... Duffy, S. (2006). Guidance on the conduct of narrative synthesis in systematic reviews: Guidance from the ESRC methods programme. Available from: http://www.lancaster.ac.uk/shm/research/nssr/research/dissemination/publications. php

Rabinowitz, A. R. \& Levin, H. S. (2014). Cognitive sequelae of traumatic brain injury. Psychiatric Clinics of North America, 37 (1), 1-11.

*Rasquin, S. M. C., Van De Sande, P., Praamstra, A. J., \& Van Heugten, C. M. (2009). Cognitive-behavioural intervention for depression after stroke: Five single case studies on effects and feasibility. Neuropsychological Rehabilitation, 19(2), 208222.

Rossiter, R., \& Holmes, S. (2013). Access all areas: creative adaptations for CBT with people with cognitive impairments - illustrations and issues. The Cognitive Behaviour Therapist, 6, $1-16$.

Scholten, A. C., Haagsma, J. A., Cnossen, M. C., Olff, M., Van Beeck, E. F., \& Polinder, S. (2016). Prevalence and risk factors of anxiety and depressive disorders following traumatic brain injury: a systematic review. Journal of Neurotrauma. 
http://doi.org/10.1089/neu.2015.4252

Scottish Intercollegiate Guidelines Network (SIGN). (2013). Brain injury rehabilitation in adults (SIGN publication no. 130). Edinburgh: SIGN. Available from URL: http://www.sign.ac.uk

Soo, C., Tate, R. L., \& Lane-Brown, A. (2011). A systematic review of acceptance and commitment therapy (ACT) for managing anxiety: Applicability for people with acquired brain injury? Brain Impairment, 12(1), 54-70.

*Tiersky, L. A., Anselmi, V., Johnston, M. V., Kurtyka, J., Roosen, E., Schwartz, T., \& DeLuca, J. (2005). A trial of neuropsychologic rehabilitation in mild-spectrum traumatic brain injury. Archives of Physical Medicine and Rehabilitation, 86(8), $1565-1574$.

Tolin,D.F. (2010) Is cognitive-bhevaioural therapy more effective than other therapies? A meta-analytic review. Clinical Psychology Review, 30 (6), 710-720.

*Tsaousides, T., Ashman, T. A., \& Gordon, W. A. (2013). Diagnosis and treatment of depression following traumatic brain injury. Brain Impairment, 14(1), 63-76.

Turner-Stokes, L., Nair, A., Sedki, I., Disler, P.B., \& Wade, D.T. (2011). Multidisciplinary rehabilitation for acquired brain injury in adults of working age. Cochrane Database of Systematic Reviews, 5, 3.

Wade, D.T., \& Halligan P. (2003). New wine in old bottles: the WHO ICF as an explanatory model of human behaviour. Clinical Rehabilitation, 17(4), 349-54.

Whiting, D.L., Simpson, G.K., McLeod, H.J., Deane, F.P. \& Ciarrochi, J. (2012). Acceptance and commitment therapy (ACT) for psychological adjustment after traumatic brain injury: reporting the protocol for a randomised controlled trial. Brain Impairment 13(3), 360 - 376. 
Williams, W.H., Evans, J.J., \& Fleminger, S. (2003).Neurorehabilitation and cognitivebehaviour therapy of anxiety disorders after brain injury: An overview and a case illustration of obsessive-compulsive disorder. Neuropsychological Rehabilitation, 13(1-2), 133-148.

Williams, W. H., Evans, J.J., \& Wilson, B.A. (2003). Neurorehabilitation for two cases of post-traumatic stress disorder following traumatic brain injury. Cognitive Neuropsychiatry, 8(1), 1-18. 
755 records identified through database

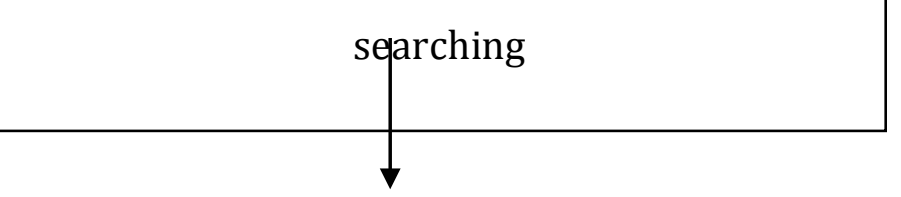

688 records after duplicates removed

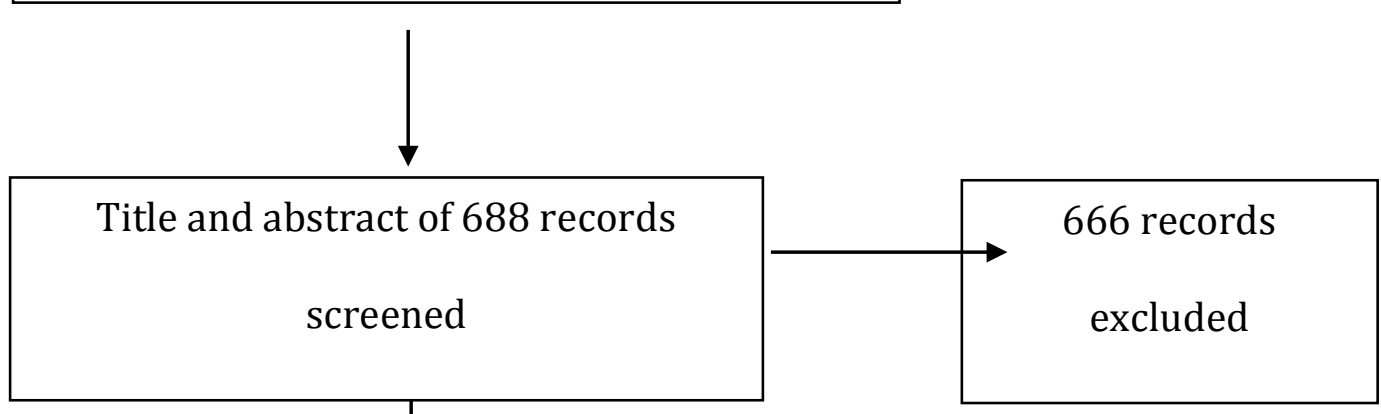

Articles assessed for eligibility:

15 review articles excluded

-23 full text review articles

Reason for exclusion:

-26 intervention study articles

-All did not provide

recommendations on

adaptations to therapy.

-Within references

of selected studies:

2 relevant

intervention studies

found.

- Articles which
-8 review articles

Selected from initial search:

8 review articles

-12 intervention studies

Selected for narrative synthesis:

- 8 review articles
14 intervention studies

excluded

Reasons for exclusion :

-3 studies related to group

CBT;

-2 discussed CBT but this was

not the form of treatment 


\section{Table 1.}

Review articles used to develop the Recommended Modification-Extraction List

\begin{tabular}{|c|c|c|c|}
\hline Study & $\begin{array}{l}\text { Stated Reason for } \\
\text { Modification to } \\
\text { Therapy }\end{array}$ & $\begin{array}{l}\text { Psychological } \\
\text { disorder/Diagno } \\
\text { sis }\end{array}$ & Main type of therapy \\
\hline Block \& West (2013) & Traumatic brain injury & - & $\begin{array}{l}\text { 'psychotherapeutic treatment' } \\
\text { including CBT, behaviour therapy, } \\
\text { CRATER therapy (a milieu/holistic- } \\
\text { based treatment which combines } \\
\text { cognitive retraining with } \\
\text { psychotherapy), narrative therapy }\end{array}$ \\
\hline Rossiter \& Holmes (2013) & $\begin{array}{l}\text { 'Cognitive } \\
\text { impairment' arising } \\
\text { from brain injury, } \\
\text { learning disabilities } \\
\text { and/or } \\
\text { neurodevelopmental } \\
\text { disorders such as ASD } \\
\text { and ADHD }\end{array}$ & - & CBT \\
\hline Tsaousides et al. (2013) & Traumatic brain injury & Depression & $\begin{array}{l}\text { CBT, behavioural interventions, } \\
\text { mindfulness training, group coping } \\
\text { skills, physical activity }\end{array}$ \\
\hline $\begin{array}{l}\text { Soo, Tate \& Lane-Brown } \\
\text { (2011) }\end{array}$ & Brain injury & Anxiety & ACT \\
\hline Broomfield et al. (2011) & Stroke & Depression & CBT \\
\hline Kangas \& McDonald (2011) & Brain injury & $\begin{array}{l}\text { 'Psychological } \\
\text { problems' }\end{array}$ & $\mathrm{ACT}, \mathrm{CBT}$ \\
\hline $\begin{array}{l}\text { Khan-Bourne \& Brown } \\
(2003)\end{array}$ & Brain injury & Depression & CBT \\
\hline Kinney (2001) & Brain injury & - & $\begin{array}{l}\text { Cognitive therapy, rational emotive } \\
\text { behaviour therapy }\end{array}$ \\
\hline
\end{tabular}


Table 2.

Modification-Extraction List, with number of intervention studies which recorded each modification to therapy.

\begin{tabular}{lcc}
\hline $\begin{array}{l}\text { Need addressed by } \\
\text { therapy }\end{array}$ & Recommended Modification & $\begin{array}{c}\text { Included in } \\
\text { modification }\end{array}$ \\
& & intervention \\
& studies (/16)
\end{tabular}

\section{Therapeutic education and formulation specific to brain injury}

Attention, concentration and alertness

Communication

Memory

Executive functioning
Include strengths and weaknesses, based on cognitive assessment, within formulation

Educate client on CBT model and treatment affect are understood Provide clear information/education on effects of brain injury in order to raise awareness and normalise common reactions

Provide breaks for rest during therapy sessions

Shorten length of sessions

(Time not specified, but assume $<50$ minutes) Increase frequency of sessions (More than once per week)

Use clear, structured questioning, and limit the use of lengthy, open-ended, or multiple questions

Incorporate visual resources into the session to enhance comprehension and draw attention to important points

Place emphasis on behavioural techniques (such as behavioural activation)

The client should have a therapy notebook or folder, review this during the session, and place important points from sessions and homework in this

Use memory aids such as written notes or audiotapes during the session - these can be reviewed between sessions

Summarise and repeat salient points at frequent intervals during the session (to refocus and help memory and learning)

Involve a family member/close friend/carer in formulation, therapy and homework tasks to enhance generalisation

Present information more slowly during session and allow extra time for response (due to slowed processing speed) 
Use summarising or an agreed-upon signal to alert the client if/when they have become tangential

Focus on concrete examples and aid clients to generate alternative solutions (due to difficulty in flexible thinking)

Therapist to take a directive and structured approach if necessary due to executive functioning/attentional deficits Model between session task completion - 'say it, show it, do it' - and encourage completion of between session tasks across a variety of situations to enhance generalisation 


\begin{tabular}{|c|c|c|c|c|c|}
\hline \multicolumn{6}{|c|}{$\begin{array}{l}\text { Table } 3 \\
\text { Summary of Intervention Studies }\end{array}$} \\
\hline $\begin{array}{l}\text { Reference } \\
\text { Diagnosis } \\
\text { Type of brain injury }\end{array}$ & Design & Sample & Treatment description & $\begin{array}{l}\text { Number } \\
\text { of } \\
\text { Adapt- } \\
\text { ations } \\
\text { from } \\
\text { marking } \\
\text { tool } \\
(/ 18)\end{array}$ & $\begin{array}{l}\text { Adapted } \\
\text { CONSORT } \\
\text { quality } \\
\text { rating } \\
(/ 6)\end{array}$ \\
\hline $\begin{array}{l}\text { D'Antonio et al. } \\
\text { (2013) } \\
\text { Depression } \\
\text { TBI }\end{array}$ & $\mathrm{RCT}$ & $\begin{array}{l}\text { CBT group }(\mathrm{N}=22) \text {, supportive psychotherapy } \\
\text { group (SPT) }(\mathrm{N}=22) \text {. } \\
\text { Average age: } 48.8 \text { years, } 26 \text { female, all } \\
\text { participants were at least } 12 \text { months post-TBI. }\end{array}$ & $\begin{array}{l}\text { Manualised treatment protocol for SPT or CBT. } \\
\text { CBT: cognitive restructuring, increasing social } \\
\text { outreach and relaxation training. } \\
\text { SPT: 'provided empathetic environment to discuss } \\
\text { issues related to depression, education about } \\
\text { depressive symptoms, and promoting the } \\
\text { individual's ability to talk about their experience, } \\
\text { without introducing specific elements of CBT'. }\end{array}$ & 3 & 3 \\
\hline $\begin{array}{l}\text { Hofer et al. (2013) } \\
\text { OCD } \\
T B I\end{array}$ & $\begin{array}{l}\text { Single } \\
\text { case }\end{array}$ & $\begin{array}{l}27 \text { year old male, severe TBI, } 3 \text { years post- } \\
\text { injury. }\end{array}$ & $\begin{array}{l}\text { Pharmacological treatment (paroxetine). } \\
\text { Prolonged exposure with response prevention ; } \\
\text { cognitive restructuring; relapse prevention. }\end{array}$ & 3 & 4 \\
\hline $\begin{array}{l}\text { Kneebone \& Jeffries } \\
\text { (2013) } \\
\text { Anxiety } \\
\text { Stroke }\end{array}$ & $\begin{array}{l}\text { Single } \\
\text { case } \\
(2)\end{array}$ & $\begin{array}{l}\text { Client 1: } 62 \text { year old male, seven months after } \\
\text { stroke. } \\
\text { Client 2: } 80 \text { year old female, one year after } \\
\text { stroke. }\end{array}$ & $\begin{array}{l}\text { Client 1: Psychoeducation, relaxation training, } \\
\text { cognitive disputation and cognitive rehabilitation. } \\
\text { Client 2: Psychoeducation, relaxation training, } \\
\text { graded exposure cognitive disputation. }\end{array}$ & 9 & 4 \\
\hline $\begin{array}{l}\text { Hsieh et al. (2012a) } \\
\text { Anxiety } \\
\text { TBI }\end{array}$ & $\begin{array}{l}\text { Single } \\
\text { case } \\
(2)\end{array}$ & $\begin{array}{l}\text { Client 1: male, "late } 40 \mathrm{~s} \text { ", severe TBI, cause of } \\
\text { injury was a fall, } 14 \text { months post-TBI. } \\
\text { Client } 2 \text { : female, "early } 30 \mathrm{~s} \text { ", severe TBI caused } \\
\text { by motor accident, } 3 \text { years } 5 \text { months post-TBI. }\end{array}$ & $\begin{array}{l}\text { Treatment based on a CBT manual developed for } \\
\text { the study including: } \\
\text { Two sessions psychoeducation regarding anxiety, } \\
\text { relaxation and slow breathing; six sessions on } \\
\text { cognitive therapy (identifying, labelling, modifying }\end{array}$ & 9 & 5 \\
\hline
\end{tabular}




\begin{tabular}{|c|c|c|c|c|c|}
\hline & & & $\begin{array}{l}\text { unhelpful thoughts) and exposure exercises; one } \\
\text { session of relapse prevention and ways of getting } \\
\text { support from others. }\end{array}$ & & \\
\hline $\begin{array}{l}\text { Hsieh et al. (2012b) } \\
\text { Anxiety } \\
\text { TBI }\end{array}$ & $\begin{array}{l}\text { Pilot } \\
\text { RCT }\end{array}$ & $\begin{array}{l}\text { Moderate or severe TBI, diagnosed with at least } \\
\text { one DSM-IV-TR anxiety disorder. } \\
\text { CBT+Motivational Interviewing group } \mathrm{N}=9 \\
\text { CBT+Non-directive counselling group } \mathrm{N}=10 \\
\text { TAU group } \mathrm{N}=8 \\
\text { Mean age }=38 \text {, mean time since } \mathrm{HI}=37.9 \text { months, } \\
\text { mean PTA }=23.1 \text { days. }\end{array}$ & $\begin{array}{l}\text { Both interventions were manualised. } \\
\text { CBT treatment included: assessment/feedback; } \\
\text { anxiety management; cognitive therapy/thinking } \\
\text { strategies; graded exposure; relapse prevention. } \\
\text { Optional elements: lifestyle balance/behavioural } \\
\text { activation; structured problem-solving, self- } \\
\text { soothing strategies. }\end{array}$ & 7 & 5 \\
\hline $\begin{array}{l}\text { Hsieh et al. (2012c) } \\
\text { Anxiety } \\
\text { TBI }\end{array}$ & $\begin{array}{l}\text { Single } \\
\text { case }\end{array}$ & $\begin{array}{l}\text { Male, early } 40 \mathrm{~s} \text {, severe TBI resulting from RTA, } \\
4 \text { months post TBI. }\end{array}$ & $\begin{array}{l}\text { Motivational Interviewing } \\
C B T \text { : psychoeducation, anxiety management, } \\
\text { cognitive therapy, graded exposure and relapse } \\
\text { prevention. }\end{array}$ & 5 & 5 \\
\hline $\begin{array}{l}\text { Kneebone \& Hull } \\
(2009) \\
\text { PTSD } \\
\text { Hydrocephalus }\end{array}$ & $\begin{array}{l}\text { Single } \\
\text { case }\end{array}$ & $\begin{array}{l}\text { Male, } 23 \text { years old, } 5 \text { months post-surgery. } \\
\text { Cognitive assessment indicated changes in } \\
\text { judgment, abstract reasoning, and ability to } \\
\text { problem-solve; did not meet full criteria for } \\
\text { PSTD according to DSM-IV; this related to } \\
\text { previous frightening hospitalisation experience } \\
\text { due to intensification of hydrocephalus }\end{array}$ & $\begin{array}{l}\text { Trauma-focused CBT including exposure, } \\
\text { cognitive disputation and relaxation training. }\end{array}$ & 3 & 4 \\
\hline $\begin{array}{l}\text { Rasquin et al. (2009) } \\
\text { Depression } \\
\text { Stroke }\end{array}$ & $\begin{array}{l}\text { Single } \\
\text { case } \\
(5)\end{array}$ & $\begin{array}{l}\text { Four female, one male, mean age }=46.2 \text { years } \\
\text { (range } 39-54 \text { ) }\end{array}$ & $\begin{array}{l}\text { Mood recording introduced and practised (1-3 } \\
\text { sessions), relaxation exercises ( } 3 \text { sessions), } \\
\text { cognitive restructuring ( } 3 \text { sessions), planning of } \\
\text { useful and enjoyable activities ( } 1 \text { session), } \\
\text { evaluating treatment ( } 1 \text { session). }\end{array}$ & 4 & 4 \\
\hline $\begin{array}{l}\text { Gracey, Oldham and } \\
\text { Kritzinger (2007) } \\
\text { Anxiety (seizure } \\
\text { related panic) } \\
\text { Stroke }\end{array}$ & $\begin{array}{l}\text { Single } \\
\text { case }\end{array}$ & $\begin{array}{l}\text { Male, } 43 \text { years old, } 20 \text { months after } \\
\text { haemorrhage. }\end{array}$ & $\begin{array}{l}\text { Collaborative formulation, cognitive restructuring, } \\
\text { behavioural experiments, relapse prevention. }\end{array}$ & 6 & 4 \\
\hline
\end{tabular}




\begin{tabular}{|c|c|c|c|c|c|}
\hline $\begin{array}{l}\text { Hodgson et al. (2005) } \\
\text { Social anxiety } \\
\text { Brain Injury }\end{array}$ & $\mathrm{RCT}$ & $\begin{array}{l}12 \text { participants in total, split into matched pairs, } \\
\text { seven males, five females. } \\
\text { CBT group } \mathrm{N}=6 \text {, mean age }=44.2 \text { years, mean } \\
\text { time since injury }=96.7 \text { months. } \\
\text { Waitlist control group } \mathrm{N}=6 \text {, mean age }=33.8 \\
\text { years, mean time since injury }=150.5 \text { months. } \\
\text { Type of brain injury: nine closed-head injury, } \\
\text { one stroke, one hypoxic brain injury, one } \\
\text { cerebral oedema. }\end{array}$ & $\begin{array}{l}\text { "Relaxation training, cognitive strategies, graded } \\
\text { exposure and assertiveness-skills training." }\end{array}$ & 9 & 2 \\
\hline $\begin{array}{l}\text { Tiersky et al. (2005) } \\
\text { Depression and } \\
\text { anxiety } \\
\text { TBI }\end{array}$ & $\mathrm{RCT}$ & $\begin{array}{l}\text { Treatment group } \mathrm{N}=11 \text {, waitlist control group } \\
\mathrm{N}=9 \text {, mild and moderate TBI. } \\
\text { Mean age for whole sample }=46.85 \text { years; } 11 \\
\text { females, } 18 \text { mild TBI, } 2 \text { moderate } \mathrm{TBI} \text {. } \\
\text { Cause: RTA }(\mathrm{N}=14) \text {, falling object }(\mathrm{N}=3) \text {, fall } \\
(\mathrm{N}=2) \text {, sports related }(\mathrm{N}=1) \text {. } \\
\text { Mean time since injury }=6.25 \text { years. }\end{array}$ & $\begin{array}{l}\text { Treatment included: } \\
\text { 1)Cognitive remedial training focusing on two } \\
\text { cognitive domains of attention and information } \\
\text { processing and memory } \\
\text { 2) CBTPhase 1: engagement. } \\
\text { Phase 2: active treatment (thought-record work, } \\
\text { behavioural experiments, cognitive rehearsal). } \\
\text { Phase 3: prevention of relapse, planning for } \\
\text { discharge. }\end{array}$ & 5 & 5 \\
\hline $\begin{array}{l}\text { Lincoln \& } \\
\text { Flannaghan (2003) } \\
\text { Depression } \\
\text { Stroke }\end{array}$ & $\mathrm{RCT}$ & $\begin{array}{l}\text { CBT group }(\mathrm{N}=39) \text {, mean age }=67.1 . \\
\text { Attention placebo }(\mathrm{N}=43) \text {, mean age }=66.1 \\
\text { years. } \\
\text { Standard care }(\mathrm{N}=41) \text {, mean age }=65.0 \\
60 \text { female, } 63 \text { male. } \\
\text { Some participants recruited } 1-3 \text { months after } \\
\text { stroke, some more than } 3 \text { months after stroke, } \\
\text { although these numbers are not specified. }\end{array}$ & $\begin{array}{l}\text { Attention placebo: conversation focusing on day- } \\
\text { to-day events and life changes. } \\
C B T \text { : manualised from Lincoln (1997) pilot study, } \\
\text { included: psychoeducation, graded task assignment, } \\
\text { activity scheduling and identification, and } \\
\text { modification of unhelpful thoughts and beliefs. }\end{array}$ & 0 & 2 \\
\hline $\begin{array}{l}\text { King (2002) } \\
\text { PTSD } \\
\text { Severe TBI with } \\
\text { dysexecutive } \\
\text { impairment }\end{array}$ & $\begin{array}{l}\text { Single } \\
\text { case }\end{array}$ & $\begin{array}{l}\text { Male, } 47 \text { years old, TBI as a result of fall and } \\
\text { being hit by a boat propeller, PTA suggestive of } \\
\text { moderate TBI, } 24 \text { months post TBI. }\end{array}$ & $\begin{array}{l}\text { Psychoeducation about PTSD and head injury, } \\
\text { anxiety-management training, systematic } \\
\text { desensitisation, and exposure to talking about the } \\
\text { accident. }\end{array}$ & 1 & 4 \\
\hline Lincoln et al. (1997) & Single & 19 participants, mean age 67.1 years. & Cognitive and behavioural techniques, including & 0 & 1 \\
\hline
\end{tabular}




\begin{tabular}{|c|c|c|c|c|c|}
\hline $\begin{array}{l}\text { Depression } \\
\text { Stroke }\end{array}$ & $\begin{array}{l}\text { case } \\
\text { series } \\
(19)\end{array}$ & $\begin{array}{l}\text { Experienced stroke mean of } 43 \text { weeks prior to } \\
\text { the study (range= } 8-109 \text { weeks). }\end{array}$ & $\begin{array}{l}\text { distraction activities, behavioural tests, graded task } \\
\text { assignments, activity scheduling, and } \\
\text { identifying/challenging negative thought patterns. }\end{array}$ & & \\
\hline $\begin{array}{l}\text { McNeill \& } \\
\text { Greenwood (1996) } \\
\text { PTSD } \\
\text { TBI }\end{array}$ & $\begin{array}{l}\text { Single } \\
\text { case }\end{array}$ & $\begin{array}{l}\text { Male, } 28 \text { years old, severe TBI as result of RTA } \\
6 \text { months post-injury. }\end{array}$ & $\begin{array}{l}\text { Phase 1: Traditional anxiety management and } \\
\text { graded exposure to avoided stimuli. } \\
\text { Phase 2: Devise an accurate account of what had } \\
\text { happened } \\
\text { Phase 3: Education about the nature of TBI, } \\
\text { specifically retrograde amnesia and post-traumatic } \\
\text { amnesia. }\end{array}$ & 1 & 3 \\
\hline $\begin{array}{l}\text { McMillan (1991) } \\
\text { PTSD } \\
\text { TBI }\end{array}$ & $\begin{array}{l}\text { Single } \\
\text { case }\end{array}$ & $\begin{array}{l}\text { Female, } 19 \text { years old, severe head injury as a } \\
\text { result of RTA, } 14 \text { months post injury. }\end{array}$ & $\begin{array}{l}\text { Cognitive-behavioural exposure techniques, } \\
\text { encouraging use of social support and confiding in } \\
\text { close family/friend. }\end{array}$ & 1 & 3 \\
\hline
\end{tabular}

Notes

Treatment/Diagnosis related abbreviations: $\mathrm{MI}=$ motivational interviewing; $\mathrm{TAU}=$ treatment as usual; $\mathrm{TBI}=$ traumatic brain injury; $\mathrm{PTA}=$ post traumatic amnesia; RTA=road traffic accident. Abbreviations of outcome measures: BDI=Beck Depression Inventory; DSM-IV-TR: Diagnostic and Statistical Manual of Mental Disorders-Fourth Edition (Text Revision);GAI=Geriatric Anxiety Index; HADS-A= Hospital Anxiety and Depression Scale- Anxiety subscale; IES=Impact of Events Scale; IES-R=Impact of Events Scale-Revised; SCLR-90= Symptoms Checklist 90-Revised; Y-BOCS: Yale-Brown Obsessive Compulsive Scale; SCID: Structured Clinical Interview for DSM-IV 